\title{
Usefulness of the Informant Questionnaire on Cognitive Decline in the Elderly for Predicting Postoperative Delirium in Elderly Patients: A Pilot Study
}

\author{
Young Ho Park, ${ }^{1,2}$ Ki-Jeong Kim,, ${ }^{3,4}$ Jin S. Yeom, ${ }^{5,6}$ SangYun Kim ${ }^{1,2}$ \\ Departments of ${ }^{1}$ Neurology, ${ }^{3}$ Neurosurgery, and ${ }^{5}$ Orthopedic Surgery, Seoul National University College of Medicine, Seoul, Korea \\ Departments of ${ }^{2}$ Neurology, ${ }^{4}$ Neurosurgery, and ${ }^{6}$ Orthopedic Surgery, Seoul National University Bundang Hospital, Seongnam, Korea
}

Background and Purpose Preexisting cognitive impairment is the strongest risk factor for delirium. We performed a pilot study to investigate whether the Informant Questionnaire on Cognitive Decline in the Elderly (IQCODE), which is a good complement to direct cognitive testing, could be useful for predicting postoperative delirium in elderly patients.

Methods Between June 2013 and May 2014, 37 patients aged 70 years or older underwent the Korean version of the Mini-Mental State Examination (K-MMSE) and completed the IQCODE (IQCODE-K) before elective spine surgery in the Spine Center at the Seoul National University Bundang Hospital. Delirium was assessed daily from the day after surgery until discharge. A Mann-Whitney U test was used to compare the K-MMSE scores and the IQCODE-K scores between the groups with and without postoperative delirium.

Results A total of three of 37 (8.1\%) patients developed delirium during their hospital stay. The K-MMSE scores were not different between the two groups $(p=0.105)$, whereas the IQCODE-K scores of patients with delirium were significantly higher than those of patients without delirium ( $p=0.021$ ), indicating greater cognitive and functional decline over the previous 10 years.

Conclusions The IQCODE may be a suitable tool for assessing preoperative cognitive function and predicting postoperative delirium in elderly patients.

Key Words delirium, surgery, aged, cognition, questionnaires.

Received: September 2, 2015 Revised: September 7, 2015 Accepted: September 7, 2015

Correspondence: SangYun Kim, MD, PhD, Department of Neurology, Seoul National University Bundang Hospital, 82 Gumi-ro 173beon-gil, Bundang-gu, Seongnam 13620, Korea

Tel: +82-31-787-7462, Fax: +82-31-787-4059, E-mail: neuroksy@snu.ac.kr

\section{INTRODUCTION}

Delirium is an acute confusional state characterized by fluctuating symptoms including inattention, disorganized thinking and an altered level of consciousness. ${ }^{1}$ Delirium occurs quite commonly as a complication of surgery in elderly patients $^{2}$ and is associated with an increased risk of morbidity and mortality as well as the loss of independence., ${ }^{3,4}$ Because preexisting cognitive impairment is the strongest risk factor for delirium, ${ }^{5}$ it is recommended that elderly patients under-

(a) This is an Open Access article distributed under the terms of the Creative Commons Attribution Non-Commercial License (http://creativecommons.org/licenses/by-nc/3.0) which permits unrestricted non-commercial use, distribution, and reproduction in any medium, provided the original work is properly cited. go brief cognitive testing at the time of admission. ${ }^{4}$ However, routine preoperative evaluation does not include the assessment of baseline cognitive function such as the Mini-Mental State Examination (MMSE) ${ }^{6}$ due to limited time and clinical resources. ${ }^{7}$ The informant-based questionnaire is a good complement to direct cognitive testing ${ }^{8}$ and is easy to administer. ${ }^{9}$ Therefore, we performed a pilot study to investigate whether the Informant Questionnaire on Cognitive Decline in the Elderly (IQCODE) ${ }_{,}^{10}$ the most widely used informant-based questionnaire, could be useful for predicting postoperative delirium in elderly patients.

\section{METHODS}

In this pilot study, we recruited 37 consecutive patients aged 
70 years or older who underwent cognitive screening before elective discectomy, laminectomy or fusion under general anesthesia in the Spine Center at the Seoul National University Bundang Hospital between June 2013 and May 2014. Patients were included in the study if they did not have a history of dementia and did not present delirium before surgery. The patient demographics and clinical profiles were obtained through a review of electronic medical records. The following two cognitive tests were performed the day before surgery: the Korean version of the MMSE (K-MMSE) ${ }^{11}$ was administered to the patients, and the Korean version of the IQCODE (IQCODE-
$\mathrm{K})^{12}$ was administered to the informants (primarily family members). The IQCODE is a 26 -item informant questionnaire that retrospectively assesses changes in cognitive and functional performance over a 10 -year period. ${ }^{10}$ Each item is rated by the relevant proxy on a 5-point scale from 1 ("much better") to 5 ("much worse"). This gives a total score of 26 to 130 that is then averaged over the 26 items to obtain a final score of 1 to 5 , in which higher scores indicate greater decline. Delirium was assessed daily by a board-certified neurologist from the day after the operation until discharge using the validated diagnostic algorithm of the confusion assessment me-

Table 1. Comparison of baseline characteristics between patients with and without postoperative delirium

\begin{tabular}{|c|c|c|c|}
\hline Characteristics & Patients with delirium $(n=3)$ & Patients without delirium $(n=34)$ & $p$ value* \\
\hline Age, median (IQR) & $78(76-80)$ & $74.5(71-78)$ & 0.245 \\
\hline Women & $3(100 \%)$ & $22(64.7 \%)$ & 0.296 \\
\hline Years of education & & & 0.658 \\
\hline $0-6$ & $2(66.7 \%)$ & $14(41.2 \%)$ & \\
\hline $7-9$ & 0 & $3(8.8 \%)$ & \\
\hline $10+$ & $1(33.3 \%)$ & $17(50.0 \%)$ & \\
\hline Prior stroke & 0 & $1(2.9 \%)$ & 0.919 \\
\hline Hypertension & $2(66.7 \%)$ & $24(70.6 \%)$ & 0.665 \\
\hline Diabetes & $1(33.3 \%)$ & $7(20.6 \%)$ & 0.530 \\
\hline Dyslipidemia & 0 & $12(35.3 \%)$ & 0.296 \\
\hline ASA score ${ }^{\dagger}$ & & & 0.637 \\
\hline 1 & 0 & $6(17.6 \%)$ & \\
\hline 2 & $3(100 \%)$ & $26(76.5 \%)$ & \\
\hline 3 & 0 & $2(5.9 \%)$ & \\
\hline Location of operation & & & 0.866 \\
\hline Cervical & 0 & $2(5.9 \%)$ & \\
\hline Thoracic & 0 & $1(2.9 \%)$ & \\
\hline Lumbosacral & $3(100 \%)$ & $31(91.2 \%)$ & \\
\hline
\end{tabular}

The values are $n(\%)$ if not indicated.

*The $p$ values were obtained using a Mann-Whitney U test or Fisher's exact test, as appropriate, ${ }^{\dagger}$ No patients had an ASA score of 4 or more. ASA: American Society of Anesthesiologists, IQR: interquartile range.
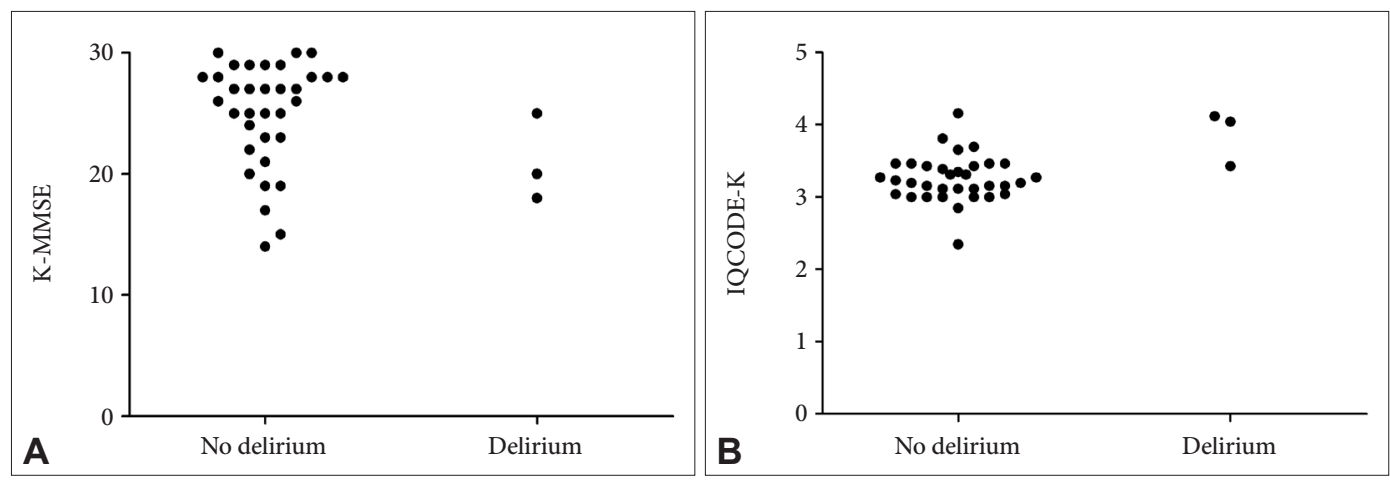

Fig. 1. Scatter plots of the cognitive profiles of patients with and without postoperative delirium. The scores of the K-MMSE (A) and IQCODE-K (B) were grouped according to whether the patient developed delirium postoperatively. The median and interquartile range of the K-MMSE scores for the groups with and without postoperative delirium were 20 (19.5-22.5) and 26.5 (23-28), respectively. The median and interquartile range of the IQCODE-K scores for the groups with and without postoperative delirium were 4.04 (3.73-4.08) and 3.21 (3.04-3.42), respectively. IQCODE-K: Korean version of the Informant Questionnaire on Cognitive Decline in the Elderly, K-MMSE: Korean Mini-Mental State Examination. 
thod. ${ }^{1}$ Baseline characteristics were compared between the groups with and without postoperative delirium using a MannWhitney U test or Fisher's exact test, as appropriate. We also compared the K-MMSE scores and the IQCODE-K scores between the two groups using a Mann-Whitney U test. All statistical analyses were performed using the PASW statistical software, version 19.0 (IBM Corp, Somers, NY, USA). The study protocol was approved by the Institutional Review Board of Seoul National University Bundang Hospital (No. B-1305/202-007).

\section{RESULTS}

A total of three of 37 (8.1\%) patients developed delirium during their hospital stay. Baseline characteristics were not different between the patients with and without delirium (Table $1)$. With respect to cognitive function, the K-MMSE scores were not different between the two groups ( $p=0.105)$, whereas the IQCODE-K scores of patients with delirium were significantly higher than those of patients without delirium ( $p=$ 0.021 ), indicating greater cognitive and functional decline over the previous 10 years (Fig. 1).

\section{DISCUSSION}

This study demonstrates that the IQCODE may be a suitable tool for assessing preoperative cognitive function and predicting delirium in elderly patients. Because the IQCODE is a questionnaire, it can be easily administered in a surgical setting. Although this measure can be affected by the informant's mood and the quality of the relationship between the informant and the patient, the IQCODE is relatively unaffected by the education level and the preoperative psychological distress of the patient. ${ }^{9}$ Despite the strength of the IQCODE, only a few studies have dealt with its usefulness for predicting postoperative delirium. ${ }^{13,14}$ The results of this pilot study have prompted us to further investigate and compare the usefulness of the IQCODE and other cognitive screening tests for predicting postoperative delirium in a larger sample size. Additionally, the development and validation of a new informant- based questionnaire that is suitable for use as a screening test for postoperative delirium is being considered.

\section{Conflicts of Interest}

The authors have no financial conflicts of interest.

\section{Acknowledgements}

This work was supported by the SNUBH Research Fund (No. 02-2013073). Dr. Jae-Won Jang and Dr. So Young Park helped with the data collection and entry.

\section{REFERENCES}

1. Inouye SK, van Dyck CH, Alessi CA, Balkin S, Siegal AP, Horwitz RI. Clarifying confusion: the confusion assessment method. A new method for detection of delirium. Ann Intern Med 1990;113:941-948.

2. Dyer CB, Ashton CM, Teasdale TA. Postoperative delirium. A review of 80 primary data-collection studies. Arch Intern Med 1995; 155:461-465.

3. Inouye SK, Rushing JT, Foreman MD, Palmer RM, Pompei P. Does delirium contribute to poor hospital outcomes? A three-site epidemiologic study. J Gen Intern Med 1998;13:234-242.

4. Inouye SK. Delirium in older persons. N Engl J Med 2006;354:11571165.

5. Cole MG. Delirium in elderly patients. Am J Geriatr Psychiatry 2004; 12:7-21.

6. Folstein MF, Folstein SE, McHugh PR. "Mini-mental state". A practical method for grading the cognitive state of patients for the clinician. J Psychiatr Res 1975;12:189-198.

7. Long LS, Shapiro WA, Leung JM. A brief review of practical preoperative cognitive screening tools. Can J Anaesth 2012;59:798-804.

8. Jorm AF. Assessment of cognitive impairment and dementia using informant reports. Clin Psychol Rev 2004;16:51-73.

9. Jorm AF. The Informant Questionnaire on cognitive decline in the elderly (IQCODE): a review. Int Psychogeriatr 2004;16:275-293.

10. Jorm AF, Jacomb PA. The Informant Questionnaire on Cognitive Decline in the Elderly (IQCODE): socio-demographic correlates, reliability, validity and some norms. Psychol Med 1989;19:1015-1022.

11. Kang Y, Na DL, Hahn S. A validity study on the Korean Mini-Mental State Examination (K-MMSE) in dementia patients. J Korean Neurol Assoc 1997; 15:300-308

12. Lee DW, Lee JY, Ryu SG, Cho SJ, Hong CH, Lee JH, et al. Validity of the Korean version of Informant Questionnaire on the Cognitive Decline in the Elderly (IQCODE). J Korean Geriatr Soc 2005;9:196-202.

13. Priner M, Jourdain M, Bouche G, Merlet-Chicoine I, Chaumier JA, Paccalin M. Usefulness of the short IQCODE for predicting postoperative delirium in elderly patients undergoing hip and knee replacement surgery. Gerontology 2008;54:116-119.

14. Wacker P, Nunes PV, Cabrita H, Forlenza OV. Post-operative delirium is associated with poor cognitive outcome and dementia. Dement Geriatr Cogn Disord 2006;21:221-227. 